\title{
Reliability of Fibronectin and P-selectin as Indicators of Vitality and Age of Wounds: An Immunohistochemical Study on Human Skin Wounds
}

\author{
Eslam S. Metwally ${ }^{1}$, Abdelmonem G. Madboly $^{1}$, Amina A. Farag', Taghreed A. Abdelaziz ${ }^{2}$, \\ Hesham A. Farag
}

\begin{tabular}{|c|c|}
\hline & ABSTRACT \\
\hline $\begin{array}{l}\text { KEYWORDS } \\
\text { Fibronectin, } \\
\text { P-selectin, } \\
\text { Immunohistochemical, } \\
\text { Wound vitality, } \\
\text { Scoring system, } \\
\text { Wound age. }\end{array}$ & $\begin{array}{l}\text { Fibronectin and P-selectin are immunohistochemical markers of skin wounds } \\
\text { vitality and age estimation. This study aimed to assess and compare between } \\
\text { fibronectin and P-selectin as markers of vitality and dating of antemortem (AM) and } \\
\text { postmortem (PM) human skin wounds. This study was carried out on } 140 \text { human } \\
\text { cases that were classified into three main groups; Group I [90 cases received at the } \\
\text { Emergency Department, Benha University Hospital, Egypt], was sub-classified } \\
\text { according to the wound age: AM1 (<30minutes; } \mathbf{n}=\mathbf{3 0}) \text {, AM2 (30-60 minutes; } n= \\
\text { 30), and AM3 (60-90 minutes; } \mathbf{n}=\mathbf{3 0} \text { ); Group II [40 cadavers received at Zenhum } \\
\text { Morgue, Ministry of Justice, Egypt], was sub-classified according to the postmortem } \\
\text { interval: PM1 ( } \leq 12 \text { hours; } \mathbf{n}=\mathbf{2 0}) \text {, PM2 }(>12 \text { hours; } \mathbf{n}=\mathbf{2 0}) \text { and Group III [10 normal } \\
\text { control skin samples]. Immunohistochemical (IHC) staining technique was } \\
\text { performed for all full thickness skin biopsies. The current study confirmed that P- } \\
\text { selectin and fibronectin were good markers of wound vitality and designed a scoring } \\
\text { system based on the increased intensity of the IHC staining in relation to wound } \\
\text { age.The current study concluded that P-selectin is more reliable and specific than } \\
\text { fibronectin as a marker of wound age estimation and vitality. }\end{array}$ \\
\hline
\end{tabular}

\section{Introduction}

Detection of wound age is defined as the time passed between the infliction of wound and cardiac arrest (if the victim is died) or wound sampling (in the living). It is always difficult to estimate a wound age with a sufficient reliability, in order to cope with the investigation of a judicial court. To make a

\footnotetext{
${ }^{(\mathbf{1})}$ Forensic Medicine \& Clinical Toxicology Department, Faculty of Medicine, Benha University, Egypt.

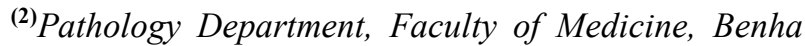
University, Egypt.

${ }^{(3)}$ Chief Medical Examiner, Forensic Medicine Authority, Ministry of Justice, Egypt.
}

valid conclusion of a wound age, systems have been developed, that can make reliable statements (Van de Goot and Fronczek, 2017).

Identification of a wound vitality and estimation of a wound age are of a paramount importance in forensic investigations (Hayashi et al., 2004). Determination of wound vitality and age depends on the process of wound healing (Balazic et al., 2005).

Many methods of detection of the vitality and age of a lesion are used such as classical histology, histochemistry, immunohistochemistry of molecular biology (Huang et al., 2007). 
Supravital reactions represent a major difficulty in estimating the wound age during the immediate post-traumatic interval (minutes to hours) (Fieguth et al., 2003).

Differential diagnosis between vital and postmortem wounds becomes difficult when the injury was inflicted very close to the time of death. Fibronectin and P-selectin are extremely early immunohistochemical markers of vitality in skin injuries (Ortiz-Rey et al., 2008).

Two of the most promising immunohistochemistry markers for vital reaction of skin wounds are fibronectin and P-selectin (Grellner and Madea, 2007).

Fibronectin is defined as a multifunctional cell adhesion protein, which is found in blood and some tissues, and forms networks in extravasation blood spots. In wound healing, it has a role in the adhesion and migration of fibroblasts, keratinocytes, and endothelial cells. Most authors consider the presence of the fibronectin network as a sign of wound vitality (Capatina et al., 2012).

The selectins (CD62) are a group of cell adhesion molecules. There are three types of selectins, all of glycoprotein structure: Eselectin in the vascular endothelium, L-selectin in leukocytes and P-selectin in platelets and endothelial cells (Capatina et al., 2015).

P-selectin (CD62p) is a 140 kilodalton $(\mathrm{kDa})$ transmembrane glycoprotein presents in megakaryocytes, activated platelets and activated endothelial cells; it is stored in the membranes of secretory granules named Weibel-Palade's bodies in the endothelium. When platelets and endothelium are activated by skin injury, the granules fuse with the cellular membrane and CD62p is expressed on the surface (Ortiz-Rey et al., 2008). Detection of CD62p could be useful in vitality determination and detection of age of the traumatic lesions, in cases with a short survival (Dressler et al., 1999).

This work aimed to: (1) assess and compare the expressions of fibronectin and Pselectin (CD62p) in human antemortem and postmortem skin wounds as markers of human skin wounds vitality as well as tools for human skin wound age estimation, using the immunohistochemical methods, (2) design a scoring system depending on the expression intensity of fibronectin and CD62p in human skin wounds, to give a skin wound a certain age with accuracy sufficient to be applied in the forensic investigations.

\section{Subjects and Methods}

\section{Subjects}

This study was carried out on 140 human cases; performed on formalin-fixed paraffinembedded skin biopsy samples which collected and processed during the period from July to December, 2017. These cases were classified into three groups, as follows:

\section{Group I (Antemortem skin wounds):}

This group included ninety human victims of accidental (cut or lacerated) vital traumatic injuries [58 males and 32 females] ranging in age from 18 to 60 years.

History taking revealed that their vital skin wounds were of different post-infliction intervals, ranging from 15 to 90 minutes. Samples were taken during routine medical emergency wound care (wound debridement) at the Emergency Department, Benha University Hospital, Egypt.

The skin wound samples of this group were classified into three sub-groups according to the wound age as follows: AM1 
(<30minutes; $\mathrm{n}=30)$, AM2 (30-60 minutes; $\mathrm{n}$ $=30)$, and AM3 (60-90 minutes; $\mathrm{n}=30)$.

Group II (Postmortem skin wounds):

Samples of this group were obtained from forty human cadavers [27 males and 13 females], ranging in age from 20-60 years, on which medico-legal autopsies were conducted at Cairo Department of Forensic Medicine (Zenhum Morgue), Ministry of Justice, Egypt. The postmortem intervals of these cadavers were within 24 hours, and the bodies were kept in cold storage at $4^{\circ} \mathrm{C}$. No obvious gross signs of putrefaction were detected at any of these cadavers.

One postmortem skin wound sample was taken from autopsy abdominal incision of each cadaver (cut wound). These postmortem skin wound samples were collected from the cadavers at fixed time post-infliction "at the beginning of autopsy".

The skin wound samples of this group were classified into two sub-groups according to the postmortem interval of the studied cadavers as follows: PMI (20 cadavers with post mortem interval $\leq 12$ hours), PM2 (20 cadavers with postmortem interval $>12$ hours).

\section{Group III (Normal control skin):}

Ten normal intact skin specimens were taken by the surgeon at Benha University Hospital, from ten patients suffering from navous lesions ( 7 females and 3 males), ranging in age from 20 to 60 years. These skin specimens represented the safety skin margins removed during excision of navous lesions.

Informed consent was taken from all cases included in groups I and III of the study; all subjects were informed about the aim, general steps and scientific value of this study. A written permission was taken from the medicolegal authority at Cairo Department of Forensic Medicine (Zenhum Morgue), Ministry of Justice, Egypt, to obtain the postmortem samples.

This research protocol was approved by the local Research Ethical board of Benha Faculty of Medicine, Benha University, Egypt. All results obtained from the subjects were registered in special sheets, which were confidential.

\section{Sampling}

Full thickness skin samples from the different studied groups were about $0.5 \times 0.5$ $\mathrm{cm}$, and $1 \mathrm{~cm}$ depth; all samples were formalinfixed paraffin-embedded blocks, and subjected to:

\section{(A) Hematoxylin and Eosin (H\& E) stain:}

From each selected formalin-fixed paraffin-embedded block; 4 micron sections were cut, and stained with conventional hematoxylin and eosin (H\& E) stain.

(B) Immunohistochemical study:

For immunohistochemical study, fibronectin, and P-selectin (CD62p) antibodies staining was performed for all biopsies using Avidin-Biotin complex techniqueto detect the presence of hemorrhage and endothelial cells of blood vessels.

For such immunohistochemical marker the steps were as follows:

1- Formalin-fixed, paraffin embedded tissue sections were cut at 3 micron sections and mounted on positively charged slides.

2- Slides were deparaffinized in 2 changes of xylene (10 minutes each) then rehydrated 
through descending grades of ethyl alcohol $(100 \%, 95 \%$, and $70 \%)$.

3- Slides were washed with distilled water three times for 2 minutes each.

4- For antigen retrieval:

a) Slides were placed in a Koplin jar containing solution of $10 \mathrm{mmol} /$ Lcitrate monohydrate buffer (PH 6.0). Koplin jar was placed in a water bath to keep a humid atmosphere inside the microwave oven.

b) Slides were placed in the microwave oven at 800 WATT power for 3 cycles, 5 minutes each. The amount of fluid in koplin jar was checked and water was added if necessary to prevent slides from drying out.

c) The jar was removed from the oven and allowed to cool for 20 minutes at room temperature.

5- Blocking endogenous peroxidase activity was done by immersing the slides in $3 \%$ hydrogen peroxide in $30 \%$ methanol for 15 minutes. Then sections were washed with distilled water to stop peroxidase activity.

6- Sections were incubated with the diluted primary antibody $(1: 100)$ for IMP3 $(0.1 \mathrm{mg} / \mathrm{ml}$ concentration, Chongqing, 400039, China) overnight.

7- Antibody-binding was detected by use of a standard labeled streptavidin-biotin system (DakoCytomation, Denmark, A/S); it was incubated with slides for 20 minutes then washed with distilled water.

8-Freshly prepared chromogen diaminobenzine (DAB) was used; it was incubated with slides for 15 minutes then washed with distilled water.

9- Slides were counter stained for 3 minutes with Mayer's hematoxylin.
10- Slides were rinsed in water, dehydrated in ascending grades of alcohol $70 \%, 95 \%$, $100 \%$ then cleared in xylene.

11- A drop of DPX wash mountant media was added and sections were covered by a glass cover.

\section{Interpretation of fibronectin expression:}

Slides were screened on low power; fibronectin expression was detected as cytoplasmic brown coloration. Immunoreactivity was assessed according to Van de Goot et al. (2014) by assessing the intensity of the staining as follows:

Score 0: None of the cells were positive for fibronectin.

Score 1: Cells express minor staining in hemorrhage as cytoplasmic positivity.

Score 2: Cells express moderate staining in hemorrhage as cytoplasmic positivity.

Score 3: Cells express strong staining in hemorrhage as cytoplasmic positivity.

Interpretation of P-selectin (CD62p) expression:

Slides were screened on low power, CD62p expression was detected as linear membranous brown coloration in endothelial cells of recently formed vascular channels. Immunoreactivity was assessed according to Van de Goot et al. (2014) by assessing the intensity of the staining as follows:

Score 0: None of the endothelial cells were positive.

Score 1: Minor endothelial cells membranous positivity.

Score 2: Moderate endothelial cells membranous positivity.

Score 3: Strong endothelial cells membranous positivity. 
Statistical design:

The collected data were tabulated and analyzed using SPSS version 16 software (Spss Inc, ILL Company, Chicago, USA). Qualitative data were presented as number and percentages, while ordinal qualitative variables were analyzed by "Linear by Linear association" test. Continuous data were expressed as mean \pm standard deviation. Differences between groups were tested using Kruskal Wallis (KW) test for nonparametric ones. Receiver Operator Characteristic (ROC) curve analysis was used to detect cutoff values for the studied scores with optimum sensitivity and specificity in prediction of wound age. Comparing areas under ROC curves (accuracy) of both markers was performed using MedCalc software (MedCalc Software, Mariakerke, Belgium). Ordinal regression analysis was used (after proved to be significant) to estimate the probabilities of wound age that was presented as mean \pm SD (probability scores). The accepted level of significance in this work was stated at $0.05(\mathrm{P}<0.05$ was considered significant) (Dawson and Trapp, 1994 and Greenberg et al., 1996).

\section{Results}

\section{Wound vitality}

The current study confirmed that Pselectin (CD62p) and fibronectin were good markers of the wound vitality, as both of them in PM skin wound samples showed a decrease in immunohistochemical (IHC) staining intensity as the PM interval increased as follows: in cadavers with an early PM interval " $\leq 12$ hours " (PM1 subgroup); most of skin wound samples exhibited mild IHC staining (score 1) for both fibronectin (55\%) and CD62p (60\%), while in cadavers with a late PM interval ">12 hours" (PM2 subgroup); most of skin wound samples exhibited a negative IHC staining (score 0 ) for both fibronectin (60\%) and CD62p (80\%), accompanied by a decrease in the mean \pm SD values of IHC scores of both of them, as illustrated in figures $(2 \& 4)$ and tables $(1 \& 2)$.

On the other hand the expression of both fibronectin and CD62p exhibited an obvious increase in the IHC staining intensity, accompanied by an increase in the mean \pm SD values of IHC scores of both of them as the wound age increased in all antemortem (AM) skin wound samples, i.e. the IHC staining of most of skin wound samples of AM1 subgroup " $<30$ minutes" was of score 1, for AM2 subgroup "30-60 minutes" the IHC staining for most of skin wound samples was of score 2 and for AM3 subgroup "60-90 minutes" it was of score 3 , as showed in figures $(1 \& 3)$ and tables ( $1 \& 2)$.

\section{Wound age estimation and scoring system:}

\section{A) As regard fibronectin:}

The majority $(60 \%)$ of control normal uninjured skin samples showed a negative IHC staining "no fibronectin staining of RBCs of hemorrhage or no hemorrhage" [score 0]. Positive staining "fibronectin staining of RBCs of hemorrhage" was recorded in all AM samples, with a highly significant $(p<0.001)$ increase in the IHC scores as follows: the majority $(60 \%)$ of skin wound samples aged $<30$ minutes (AM1 subgroup) showed mild staining [score 1], the majority $(73 \%)$ of skin wound samples aged 30-60 minutes (AM2 subgroup) showed moderate staining [score 2], while the vast majority $(80 \%)$ of skin wound samples aged 60-90 minutes (AM3 subgroup) showed strong staining [score 3], as illustrated in figures $(1 \& 3)$ and table (1). 
B) As regard P-selectin (CD62p):

In $50 \%$ of control normal uninjured skin samples CD62p showed mild staining of blood vessel endothelial cells [score 1]. Also positive staining of blood vessel endothelial cells was recorded in all AM samples, with a highly significant $(p<0.001)$ increase in the IHC scores as follows: the majority $(80 \%)$ of skin wound samples aged $<30$ minutes (AM1 subgroup) showed mild staining [score 1], the majority $(90 \%)$ of skin wound samples aged 30-60 minutes (AM2 subgroup) showed moderate staining [score 2], while the vast majority $(96.7 \%)$ of skin wound samples aged 60-90 minutes (AM3 subgroup) showed strong staining [score 3], as illustrated in figures $(1 \&$ 3 ) and table (1).

\section{The probability of scoring system:}

To set up a reliable and an easy guideline to help the forensic pathologist to identify the wound sample origin i.e. AM or PM wound and to estimate the AM skin wound age, the present study statistically measured the probability of the designed scoring system:

\section{A) As regard fibronectin:}

If the IHC score of the skin wound sample was 0 ; the probability that the origin of the sample was from an AM skin wound aged $<30$ minutes or it was from a PM skin wound of a cadaver with a PM interval $>12$ hours was $43 \%$ for both. If the IHC score was 1 ; the probability that the origin of the sample was from an AM skin wound of age $<30$ minutes was $41 \%$ or it was from a PM skin wound of a cadaver with a PM interval $\leq 12$ hours was $25 \%$. If the IHC score was 2; the probability that the origin of the sample was from an AM skin wound aged 30-60 minutes was $67 \%$. But if the
IHC score was 3 ; the probability that the origin of the sample was from an AM skin wound aged $60-90$ minutes was as high as $96 \%$, as illustrated in table (3).

\section{B) As regard P-selectin (CD62p):}

If the IHC score of the skin wound sample was 0 ; the probability that the origin of the sample was from a PM skin wound of a cadaver with a PM interval $>12$ hours was $53 \%$ or it was from an AM skin wound aged $<30$ minutes was $20 \%$. If the IHC score of the skin wound sample was 1 ; the probability that the origin of the sample was from an AM skin wound aged $<30$ minutes was $56 \%$ or it was from a PM skin wound of a cadaver with a PM interval $\leq 12$ hours was $28 \%$. If the IHC score was 2 ; the probability that the origin of the sample was from an AM skin wound of age 30-60 minutes was $96 \%$. If the IHC score was 3 ; the probability that the origin of the sample was from an AM skin wound aged 60-90 minutes was as high as $100 \%$, as illustrated in table (3).

A receiver operator characteristic (ROC) curve was conducted to compare between fibronectin and CD62p sensitivity and specificity in determining wound vitality and wound age, and the current study confirmed that in case of fibronectin IHC score 3; the probability that the skin wound sample was from a PM skin wound was $0 \%$. On the other hand, as regard $\mathrm{CD} 62 \mathrm{p}$ the current study confirmed that in case of an IHC score 2 or 3 ; the probability that the skin sample was from a $\mathrm{PM}$ skin wound was $0 \%$, indicating that $\mathrm{P}$ selectin (CD62p) is more reliable and specific than fibronectin as a marker of wound vitality and wound age estimation, as illustrated in figures (5 \& 6) and tables (3, 4 \& 5). 

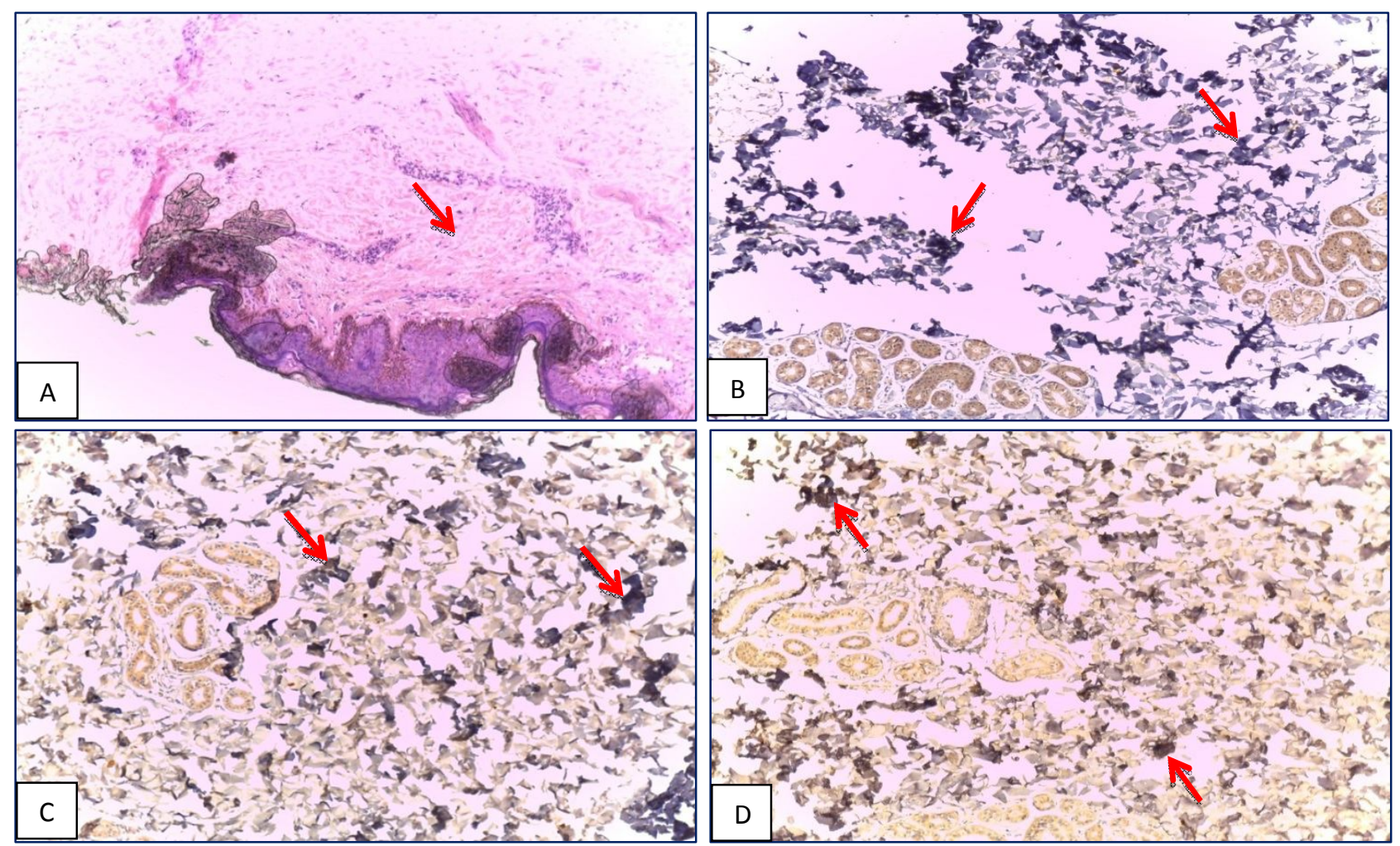

Fig. (1): A photomicrograph illustrating fibronectin immunohistochemical (IHC) staining of different studied skin samples: (A) a section of normal uninjured skin of control group, showing no hemorrhage (score 0); (B) a section of an antemortem skin wound $[<30$ minutes] exhibiting mild fibronectin staining of hemorrhage (score 1); (C) a section of an antemortem skin wound [30-60 minutes] showing moderate fibronectin staining of hemorrhage (score 2); (D) a section of an antemortem skin wound [60 -90 minutes] exhibiting strong fibronectin staining of hemorrhage (score 3) [red arrows], streptavidin-biotin technique (DAB x200).
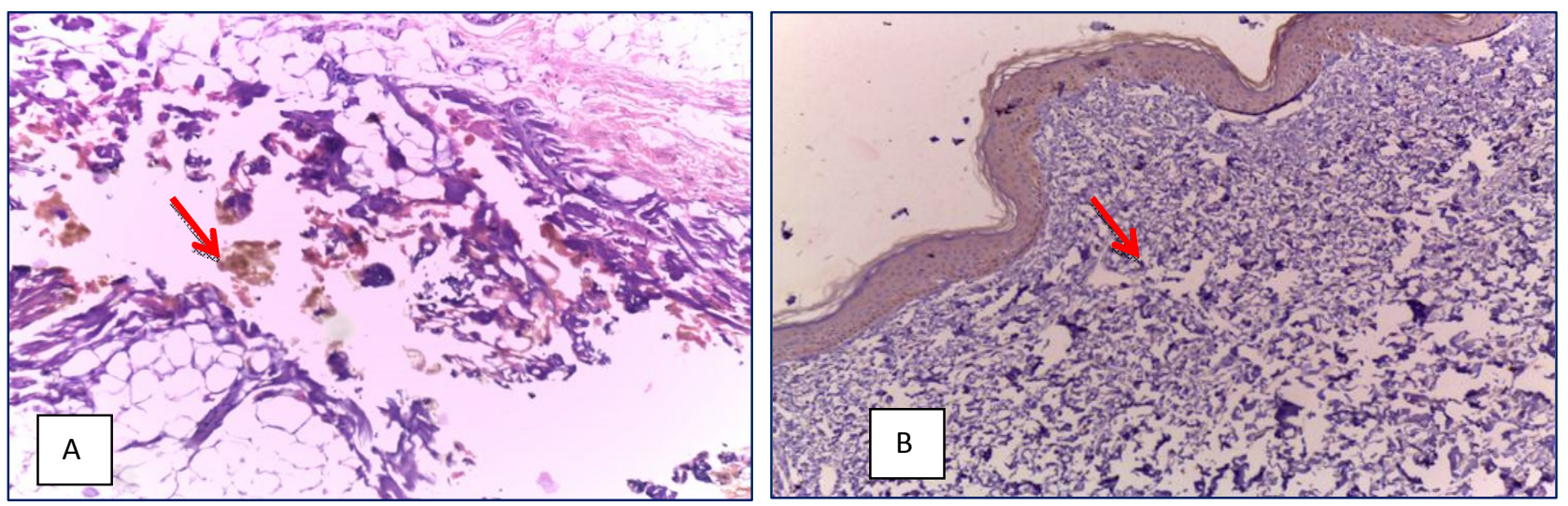

Fig. (2): A photomicrograph illustrating fibronectin IHC staining of different studied postmortem (PM) skin samples: (A) a section of a PM skin wound [early PM interval " $<12$ hours"] exhibiting mild fibronectin staining of hemorrhage (score 1); (B) a section of a PM skin wound [late PM interval " $>12$ hours"] showing negative fibronectin staining of hemorrhage (score 0) [red arrows] streptavidinbiotin technique (DAB x200). 


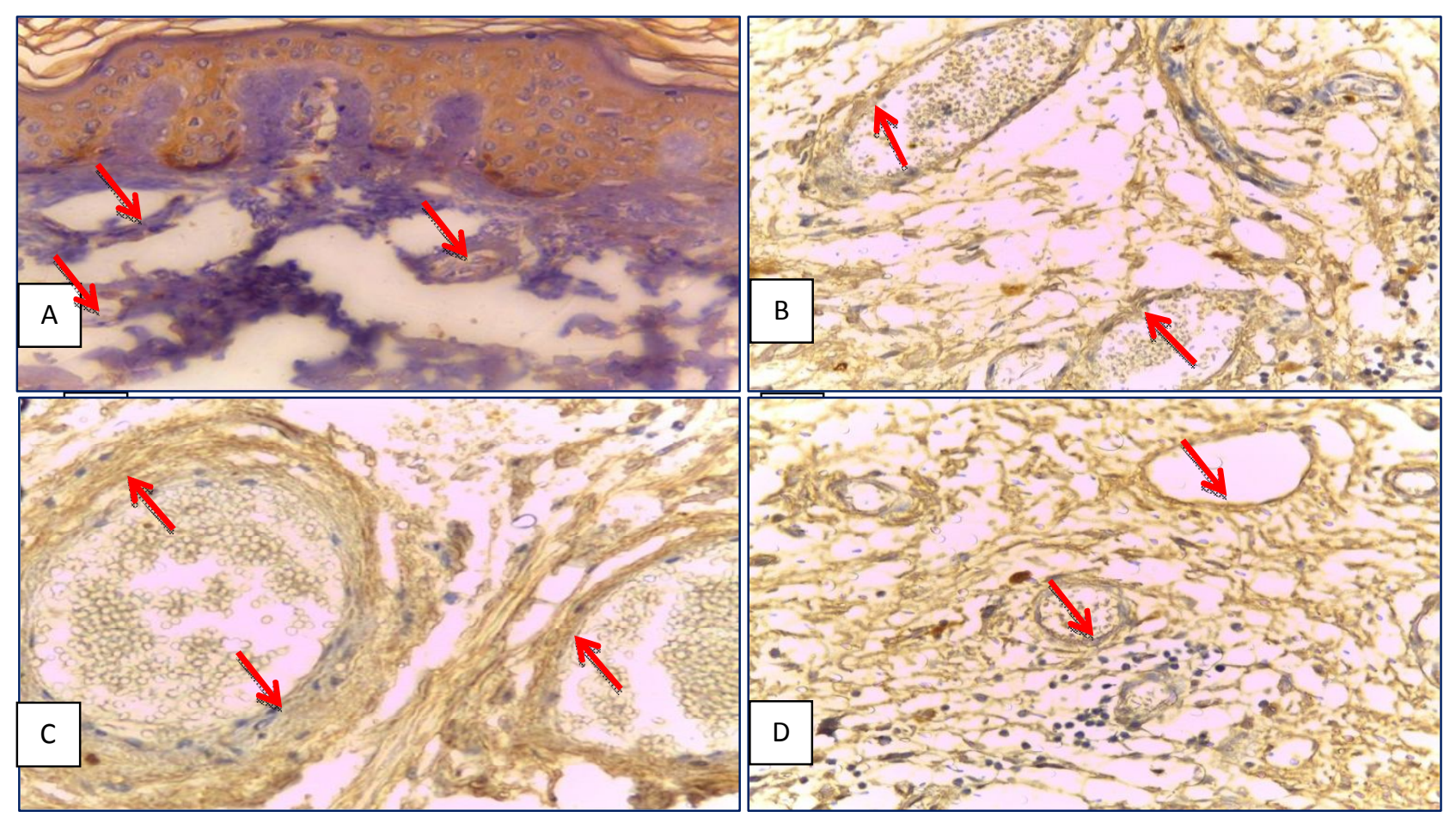

Fig. (3): A photomicrograph illustrating P-selectin (CD62p) IHC staining of different studied skin samples: (A) a section of normal uninjured skin of control group, showing mild CD62p staining of blood vessel endothelial cells (score 1); (B) a section of an antemortem skin wound $[<30$ minutes] showing mild CD62p staining of blood vessels endothelial cells (score 1); (C) a section of an antemortem skin wound [30 - 60 minutes] exhibiting moderate CD62p staining of blood vessels endothelial cells (score 2); (D) a section of an antemortem skin wound [60 - 90 minutes] showing strong CD62p staining of blood vessels endothelial cells (score 3) [red arrows], streptavidin-biotin technique (DAB x400).
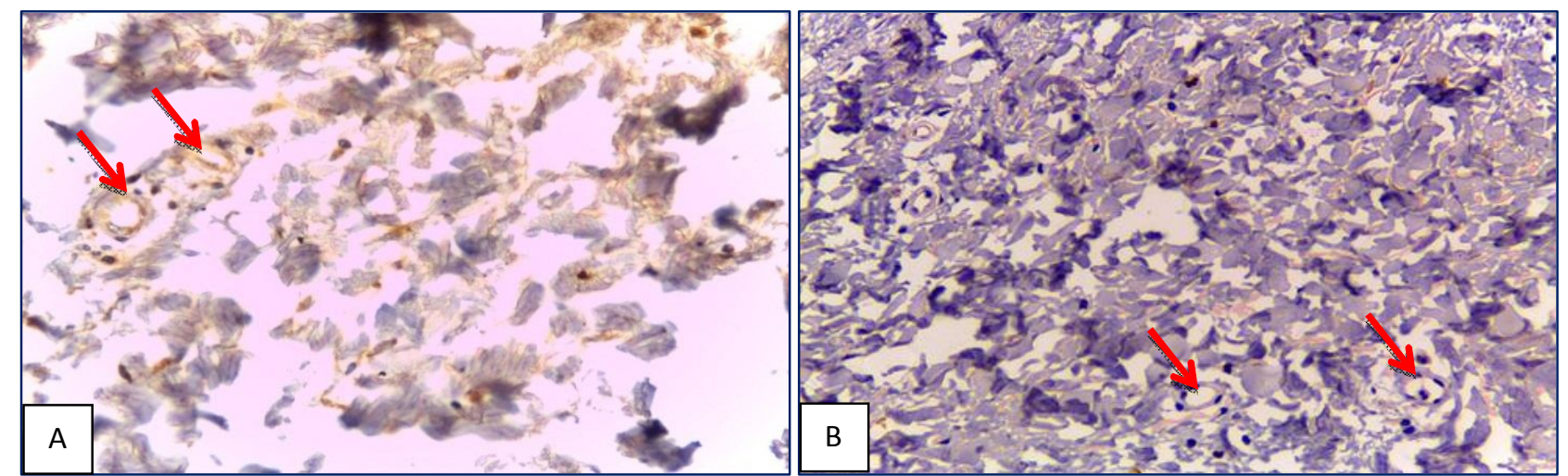

Fig. (4): A photomicrograph illustrating P-selectin (CD62p) IHC staining of different studied postmortem (PM) skin samples: (A) a section of a PM skin wound [early PM interval " $<12$ hours"] showing mild CD62p staining of blood vessels endothelial cells (score 1); (B) a section of a PM skin wound [late PM interval " $>12$ hours"] showing negative CD62p staining of blood vessel endothelial cells (score 0 ) [red arrows], streptavidin-biotin technique (DAB x400). 
Table (1): Number and percentage of the immunohistochemical (IHC) scores of fibronectin and P-selectin (CD62p) expressions, among the different studied wound samples

\begin{tabular}{|c|c|c|c|c|c|c|c|c|c|c|c|}
\hline \multirow{2}{*}{\multicolumn{2}{|c|}{ Groups }} & \multicolumn{5}{|c|}{ Fibronectin } & \multicolumn{5}{|c|}{ P-selectin (CD62p) } \\
\hline & & \multirow{2}{*}{$\begin{array}{c}\text { Score } 0 \\
12\end{array}$} & \multirow{2}{*}{$\begin{array}{c}\text { Score1 } \\
18\end{array}$} & \multirow{2}{*}{$\begin{array}{c}\text { Score2 } \\
0\end{array}$} & \multirow{2}{*}{$\begin{array}{c}\text { Score3 } \\
0\end{array}$} & \multirow{2}{*}{$\begin{array}{c}\text { Total } \\
30\end{array}$} & \multirow{2}{*}{$\begin{array}{c}\text { Score0 } \\
6\end{array}$} & \multirow{2}{*}{$\begin{array}{c}\text { Score1 } \\
24\end{array}$} & \multirow{2}{*}{$\begin{array}{c}\text { Score2 } \\
0\end{array}$} & \multirow{2}{*}{$\begin{array}{c}\text { Score3 } \\
0\end{array}$} & \multirow{2}{*}{$\begin{array}{c}\text { Total } \\
30\end{array}$} \\
\hline & $\mathbf{n}$ & & & & & & & & & & \\
\hline & $\%$ & $40.0 \%$ & $60.0 \%$ & $0.0 \%$ & $0.0 \%$ & $100.0 \%$ & $20.0 \%$ & $80.0 \%$ & $0.0 \%$ & $0.0 \%$ & $100.0 \%$ \\
\hline \multirow{2}{*}{ AM2 } & $\bar{n}$ & $\overline{0}$ & $\overline{7}$ & 22 & $\overline{1}$ & 30 & 0 & 3 & 27 & 0 & 30 \\
\hline & $\%$ & $0.0 \%$ & $23.3 \%$ & $73.3 \%$ & $3.3 \%$ & $100.0 \%$ & $0.0 \%$ & $10.0 \%$ & $90.0 \%$ & $0.0 \%$ & $100.0 \%$ \\
\hline \multirow{2}{*}{ AM3 } & $\mathbf{n}$ & 0 & 0 & 6 & 24 & 30 & 0 & 0 & 1 & 29 & 30 \\
\hline & $\%$ & $0.0 \%$ & $0.0 \%$ & $20.0 \%$ & $80.0 \%$ & $100.0 \%$ & $0.0 \%$ & $0.0 \%$ & $3.3 \%$ & $96.7 \%$ & $100.0 \%$ \\
\hline \multirow{2}{*}{ PM1 } & $\mathbf{n}$ & 4 & 11 & 5 & 0 & 20 & 8 & 12 & 0 & 0 & 20 \\
\hline & $\%$ & $20.0 \%$ & $55.0 \%$ & $25.0 \%$ & $0.0 \%$ & $100.0 \%$ & $40.0 \%$ & $60.0 \%$ & $0.0 \%$ & $0.0 \%$ & $100.0 \%$ \\
\hline \multirow{2}{*}{ PM2 } & $\bar{n}$ & 12 & 8 & 0 & 0 & 20 & 16 & 4 & 0 & 0 & 20 \\
\hline & $\%$ & $60.0 \%$ & $40.0 \%$ & $0.0 \%$ & $0.0 \%$ & $100.0 \%$ & $80.0 \%$ & $20.0 \%$ & $0.0 \%$ & $0.0 \%$ & $100.0 \%$ \\
\hline \multirow{2}{*}{ Control } & $\bar{n}$ & 6 & 4 & 0 & 0 & 10 & 5 & 5 & 0 & 0 & 10 \\
\hline & $\%$ & $60.0 \%$ & $40.0 \%$ & $0.0 \%$ & $0.0 \%$ & $100.0 \%$ & $50.0 \%$ & $50.0 \%$ & $0.0 \%$ & $0.0 \%$ & $100.0 \%$ \\
\hline \multirow{2}{*}{ Total } & n & 34 & 48 & 33 & 25 & 140 & 35 & 48 & 28 & 29 & 140 \\
\hline & $\%$ & $24.3 \%$ & $34.3 \%$ & $23.6 \%$ & $17.9 \%$ & $100.0 \%$ & $25.0 \%$ & $34.3 \%$ & $20.0 \%$ & $20.7 \%$ & $100.0 \%$ \\
\hline
\end{tabular}

$\mathrm{n}$ : number, for fibronectin: liner by linear association $=4.57, \mathrm{p}=0.032(\mathrm{~S}), \mathrm{AM}=$ antemortem, For CD62 $\mathrm{p}$ : liner by linear association $=12.4, \mathrm{p}=0.001(\mathrm{HS}), \mathrm{PM}=$ postmortem

Table (2): Mean \pm SD of the immunohistochemical (IHC) scores of fibronectin and P-selectin (CD62p) expressions among the different studied wound samples

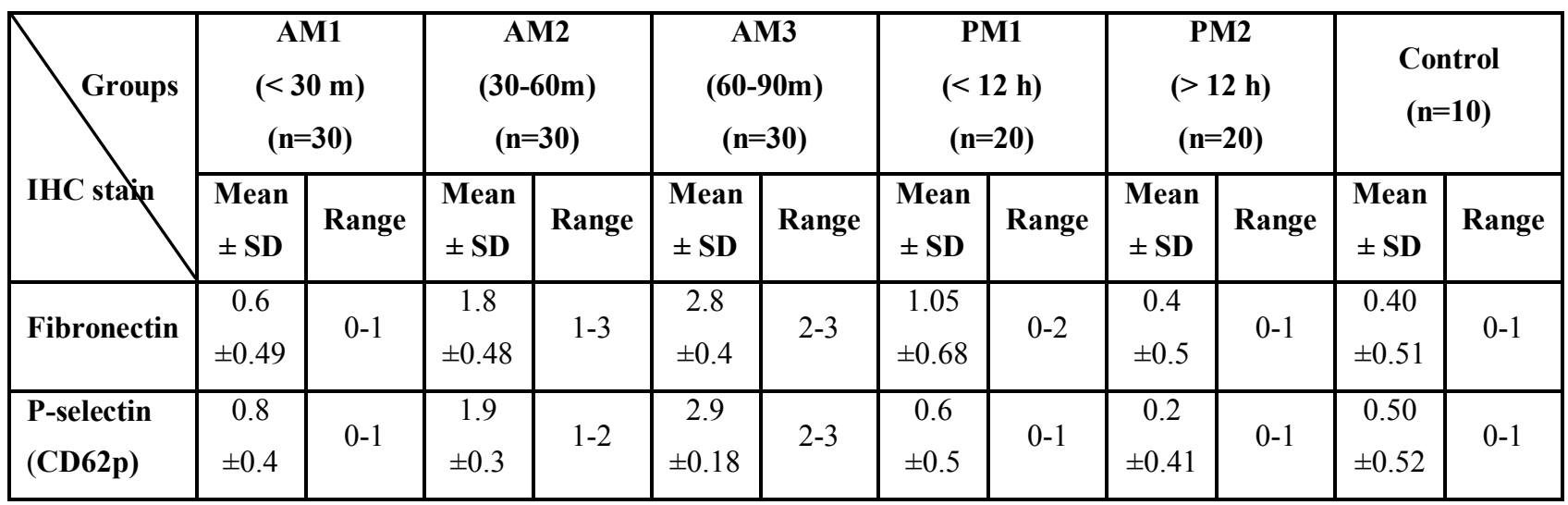

$\mathrm{n}:$ number, Kruskal Wallis $[\mathrm{KW}]$ test $\rightarrow$ for fibronectin=104.1, for CD62p =118, AM= antemortem, $\mathrm{p}<0.001$ (HS) for both fibronectin and CD62p, $\mathrm{PM}=$ postmortem, $\mathrm{SD}=$ standard deviation 
Table (3): Probabilities of wound age according to the immunohistochemical (IHC) scores of fibronectin and P-selectin (CD62p), [based on ordinal regression], among different studied wound samples

\begin{tabular}{|c|c|c|c|c|c|c|}
\hline \multirow{2}{*}{\multicolumn{2}{|c|}{$\begin{array}{c}\text { Scores } \\
\text { (explanatory } \\
\text { variables) }\end{array}$}} & \multicolumn{5}{|c|}{ Probability of wound age (Outcome variable, Mean \pm SD) } \\
\hline & & \multirow{2}{*}{$\begin{array}{c}\text { AM1 wound } \\
(<\mathbf{3 0} \mathbf{~ m}) \\
0.43 \pm 0.50\end{array}$} & \multirow{2}{*}{$\begin{array}{c}\text { AM2 wound } \\
\text { (30-60m) } \\
0.0 \pm 0.00\end{array}$} & \multirow{2}{*}{$\begin{array}{c}\text { AM3 wound } \\
\text { (>60-90 m) } \\
0.0 \pm 0.0\end{array}$} & \multirow{2}{*}{$\begin{array}{c}\text { PM1 wound } \\
(<\mathbf{1 2} \text { h }) \\
0.14 \pm 0.17\end{array}$} & \multirow{2}{*}{$\begin{array}{c}\begin{array}{c}\text { PM2 wound } \\
(>\mathbf{1 2})\end{array} \\
0.43 \pm 0.39\end{array}$} \\
\hline \multirow{4}{*}{ 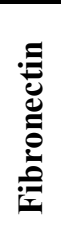 } & Score 0 & & & & & \\
\hline & Score 1 & $0.41 \pm 0.45$ & $0.16 \pm 0.31$ & $0.0 \pm 0.0$ & $0.25 \pm 0.31$ & $0.18 \pm 0.24$ \\
\hline & Score 2 & $0.00 \pm 0.00$ & $0.67 \pm 0.44$ & $0.18 \pm 0.35$ & $0.15 \pm 0.36$ & $0.0 \pm 0.0$ \\
\hline & Score 3 & $0.0 \pm 0.0$ & $0.04 \pm 0.19$ & $0.96 \pm 0.19$ & $0.0 \pm 0.0$ & $0.0 \pm 0.0$ \\
\hline \multicolumn{2}{|c|}{ p value } & 0.7 & $<0.001 *$ & $<0.001 *$ & $<0.001 *$ & $<0.001 *$ \\
\hline \multirow{4}{*}{ ڤి } & Score 0 & $0.20 \pm 0.40$ & $0.0 \pm 0.0$ & $0.0 \pm 0.0$ & $0.27 \pm 0.22$ & $0.53 \pm 0.32$ \\
\hline & Score 1 & $0.56 \pm 0.45$ & $0.07 \pm 0.15$ & $0.0 \pm 0.0$ & $0.28 \pm 0.38$ & $0.09 \pm 0.17$ \\
\hline & Score 2 & $0.0 \pm 0.0$ & $0.96 \pm 0.02$ & $0.04 \pm 0.02$ & $0.0 \pm 0.0$ & $0.0 \pm 0.0$ \\
\hline & Score 3 & $0.0 \pm 0.0$ & $0.0 \pm 0.0$ & $1.0 \pm 0.0001$ & $0.0 \pm 0.0$ & $0.0 \pm 0.0$ \\
\hline \multicolumn{2}{|c|}{ p value } & $<0.001 *$ & $<0.001 *$ & $<0.001 *$ & $<0.001 *$ & $<0.001 *$ \\
\hline
\end{tabular}

$\mathrm{AM}=$ antemortem, $\mathrm{PM}=$ postmortem, $\mathrm{SD}=$ standard deviation, Kruskal Wallis $[\mathrm{KW}]$ test was used to compare probabilities, Probabilities were calculated by ticking the estimated response probabilities box in ordinal regression model , * significant

Table (4): Cutoff values, AUC and CI of ROC curve for comparing accuracy of fibronectin and P-selectin (CD62p) immunohistochemical scores in prediction of antemortem wounds

\begin{tabular}{|l|c|c|c|c|c|c|c|}
\hline \multicolumn{1}{|c|}{ Score cutoff } & $\begin{array}{c}\text { Sensitivity } \\
\mathbf{\%}\end{array}$ & $\begin{array}{c}\text { Specificity } \\
\mathbf{\%}\end{array}$ & $\begin{array}{c}\text { PPV } \\
\mathbf{\%}\end{array}$ & $\begin{array}{c}\text { NPV } \\
\mathbf{\%}\end{array}$ & $\begin{array}{c}\text { Accuracy } \\
\mathbf{\%}\end{array}$ & AUC & $\mathbf{9 5 \%}$ CI \\
\hline Fibronectin score $\geq \mathbf{2}$ & $59 \%$ & $90 \%$ & $91.4 \%$ & $54.9 \%$ & $70 \%$ & 0.789 & $0.72-0.86$ \\
\hline CD62p score $\geq \mathbf{2}$ & $63.3 \%$ & $100 \%$ & $100 \%$ & $60.2 \%$ & $76.4 \%$ & 0.882 & $0.83-0.94$ \\
\hline \multicolumn{7}{|c|}{ p value was < $\mathbf{0 . 0 0 1}$} \\
\hline
\end{tabular}

$\mathrm{PPV}=$ positive predictive value; $\mathrm{NPV}=$ negative predictive value; $\mathrm{CI}=$ confidence interval, $\mathrm{AUC}=$ area under the curve; ROC curve $=$ receiver operator characteristic curve

Table (5): Cutoff values, AUC and CI of ROC curve for comparing accuracy of fibronectin and P-selectin (CD62p) immunohistochemical scores in prediction of postmortem wounds

\begin{tabular}{|l|c|c|c|c|c|c|c|}
\hline \multicolumn{1}{|c|}{ Score cutoff } & Sensitivity\% & Specificity\% & $\begin{array}{c}\text { PPV } \\
\%\end{array}$ & $\begin{array}{c}\text { NPV } \\
\%\end{array}$ & $\begin{array}{c}\text { Accuracy } \\
\%\end{array}$ & AUC & $\mathbf{9 5 \%}$ CI \\
\hline Fibronectin score $\leq \mathbf{1}$ & $40 \%$ & $82 \%$ & $47.1 \%$ & $77.3 \%$ & $70 \%$ & 0.733 & $0.65-0.82$ \\
\hline CD62p score $\leq \mathbf{1}$ & $60 \%$ & $91 \%$ & $72.8 \%$ & $85 \%$ & $82.1 \%$ & 0.869 & $0.81-0.93$ \\
\hline \multicolumn{7}{|c|}{ p value was $<\mathbf{0 . 0 0 1}$} \\
\hline
\end{tabular}

$\mathrm{PPV}=$ positive predictive value; $\mathrm{NPV}=$ negative predictive value; $\mathrm{CI}=$ confidence interval, $\mathrm{AUC}=$ area under the curve; ROC curve $=$ receiver operator characteristic curve 


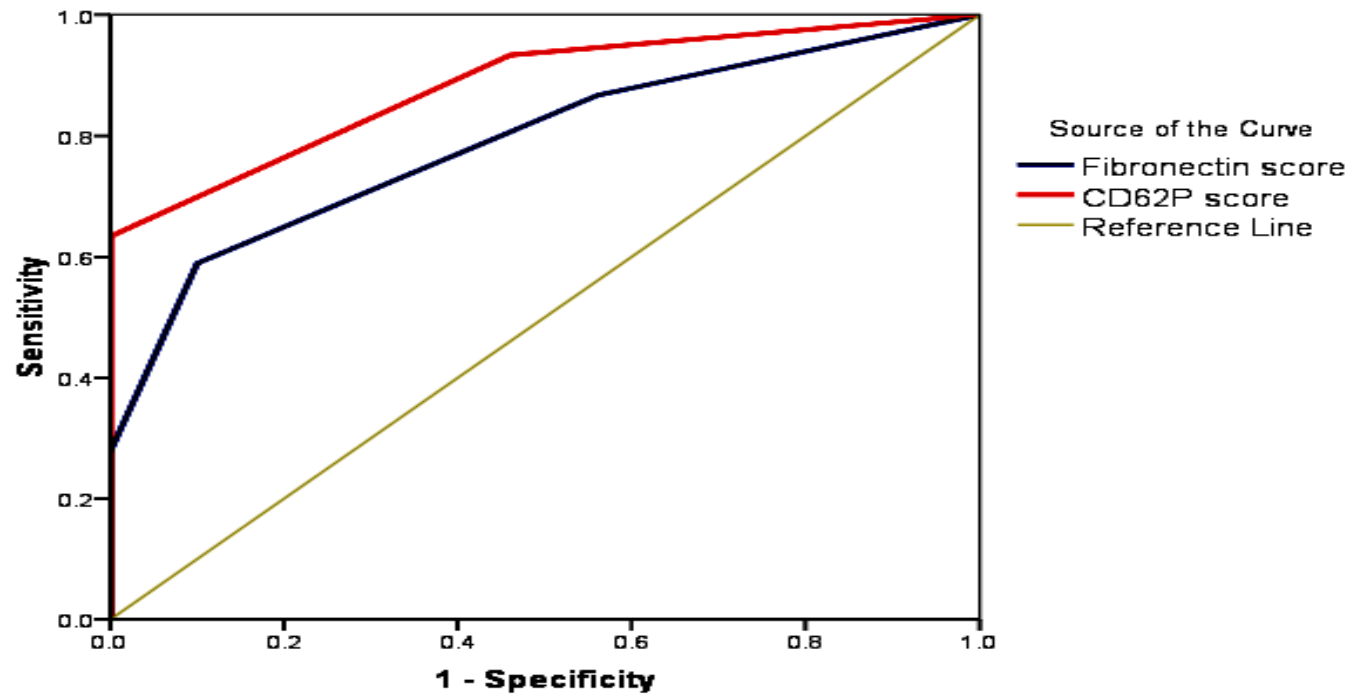

Fig. (5): Receiver Operator Characteristic (ROC) curve for comparing accuracy of fibronectin and P-selectin (CD62p) immunohistochemical scores in prediction of antemortem wounds.

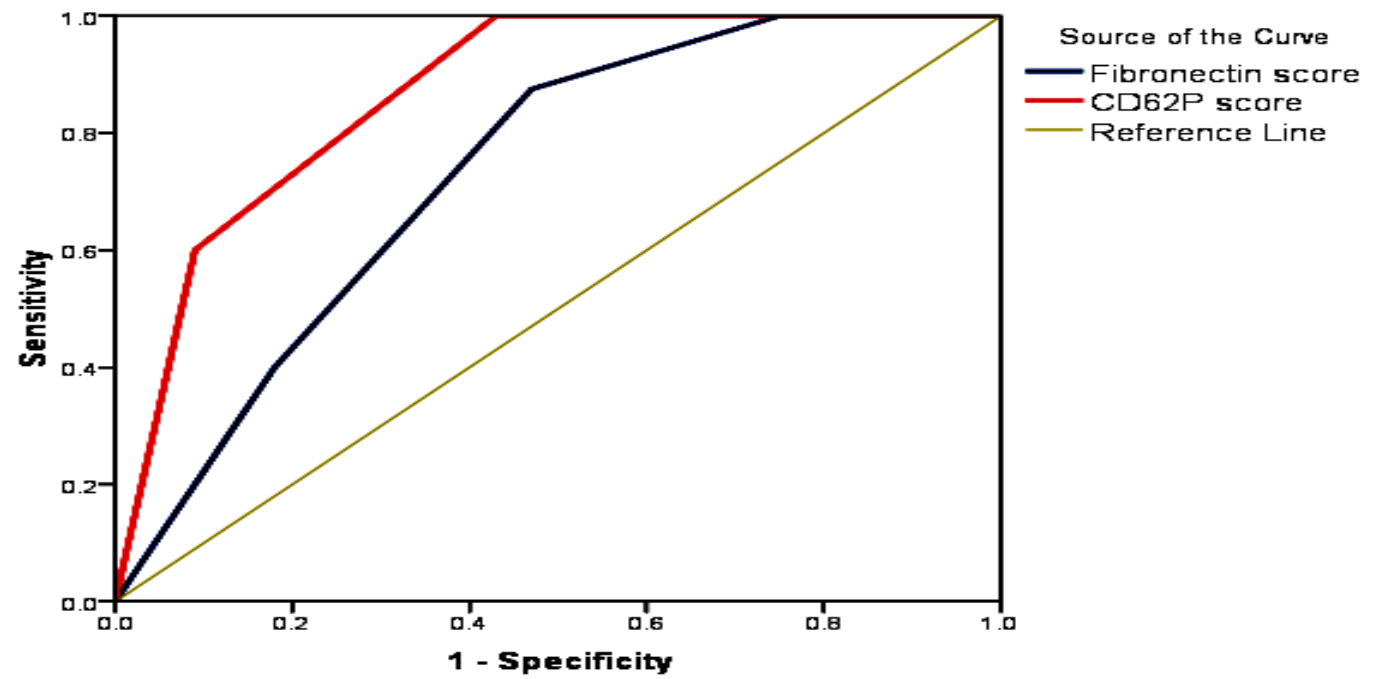

Fig. (6): Receiver Operator Characteristic (ROC) curve for comparing accuracy of fibronectin and P-selectin (CD62p) immunohistochemical scores in prediction of postmortem wounds. 


\section{Discussion}

Estimation of wound age remains a major concern in forensic investigations. The forensic pathologist has to differentiate between antemortem (AM) and postmortem (PM) wounds, as well as try to determine the survival time of the injured person(Ishida et al., 2009 and Casse et al., 2016).

The main obstacle of postmortem studies - other than decomposition process is the uncertainty of exact survival time (wound age). Therefore, strictly selected cases are required with well documented wound infliction times (Grellner and Madea, 2007).

Van de Goot et al. (2014) stated that the initiation of the coagulation cascade proteins: fibronectin, P-selectin (CD62p) and factor VIII in wound hemorrhage, are useful tools in estimating the wound age, particularly in recent wounds.

The current study illustrated that Pselectin (CD62p) and fibronectin were good markers of the wound vitality, as both of them in PM skin wound samples showed a decrease in IHC staining intensity as the PM interval increased. While their IHC expressions exhibited an obvious increase in the IHC staining intensity, as the wound age increased in all AM skin wound samples.

Ceauşu and Capatina (2014) found in their study that the IHC expression of fibronectin and P-selectin at the level of the extracellular matrix, fibroblasts and capillaries is useful in assessing the vitality of traumatic lesions.

An old study conducted by Betz et al. (1992) revealed a positive reaction for fibronectin in vital human skin wounds at an interval of more than 30 minutes after injury infliction and a faint focal positive reaction for postmortem lesions, and they proposed that only wounds which showed strong positive fibronectin staining should be regarded as vital.

In a study carried out on animals on which wounds have been inflicted within 0-5 minutes after cardiac arrest, occurrence of fibronectin network in some of the cases was observed (Grellner et al., 1998), but another study pointed to this as a false-positive reaction due to spravital reactions (Fieguth et al., 1997).

Ortiz-Rey et al. (2003) stated that postmortem expression of fibronectin was due to passive release of serum fibronectin from damaged blood vessels following death.

Dressler et al.(2002) in their studies on human and experimental animal skin specimens found that P-selectin immunopositivity starts as early as 3 minutes and lasts for seven hours.

Another study on human skin injuries observed immunopositivity of CD62p in endothelial cells after one minute with a steady increase after 10- 15 minutes (Cecchi et al., 2004).

Ortiz-Rey et al. (2008) stated that CD62p immunopositivity in vascular endothelial cells in post-mortem wounds obtained 6 hours after death, was due to the supravital phenomenon.

There are two distinct processes that may occur in the early PM period:

a) passive extravasation of blood from damaged vessels (independent of cardiac activity), associated with the advent of blood infiltrates;

b) early stages of inflammation and tissue repair (independent of both cardiac and brain activity), explained by the fact that death is a process and not an event (Capatina et al., 2015).

On the other hand Capatina et al. (2013) concluded that for forensic practice the 
usefulness of fibronectin and P-selectin is debatable, as both of them have a moderate sensitivity and low specificity. If they used in detecting the vitality and age of the wound, there is a need for a much larger scale study that must take into consideration factors like CPR, individual pathologies, and so on.

Moreover, Cecchi (2010) listed the markers which should not be used as they are unreliable as: prostaglandins, fibrin, cytokeratin $13, \alpha 1$-anti-chymotrypsin, $\alpha 2-$ macroglobulin, histamine, proteases, ICAM-1 and selectin L.

The present study confirmed that both fibronectin and P-selectin (CD62p) can be used as markers in determining the wound age in AM skin wound samples, as there is increase in IHC intensity in relation to time passed since skin wound infliction, and a scoring system was designed for this increase in the intensity of the IHC staining in relation to time passed since skin wound inflection (wound age).

In AM human skin wounds, fibronectin appears in about 10-20 minutes and is still present after 17 days (Betz et al., 1992). In a study of AM human skin wounds done by Capatina et al. (2013) fibronectin was constantly present, and the intensity of the staining was independent upon the estimated wound age, but this results was notstatistically significant.

P-selectin in AM human skin wound samples, appears in minutes and disappears in about seven hours (Dressler et al., 2000 \& Kondo, 2007). In Capatina et al. (2013) study, $\mathrm{P}$-selectin was constantly present, irrespective of the survival time. However, the most intense staining was identified in wounds of about 30 minutes age.

In Balazic et al.(2005) study a semiquantitative score for evaluating fibronectin reaction was done as follows $[0=$ no reaction;
$1=$ mild staining; $2=$ moderate staining and $3=$ intense staining], while Van de Goot and Fronczek (2017) described a fibronectin IHC scoring as follows $[(1)=$ slight staining; $(2)=$ clear staining and (3) = massive staining], and a CD62p IHC scoring as follows $[(1)=$ slight linear endothelial staining; (2) = obvious linear staining and intravascular staining and (3) = strong extra vascular staining].

The present work statistically measured the probability of the designed scoring system as mentioned in details in the results. Van de Goot et al. (2014) found that if IHC score was 0 , the probability of uninjured skin sample was the highest $(87 \%$ for fibronectin and $88 \%$ for $\mathrm{P}$ selectin). If IHC score was 1 or 2 , the probabilities of AM wound aged few minutes were the highest $(82 / 90 \%$ for fibronectin and $82 / 83 \%$ for P-selectin). If IHC score was 3 , the probability of AM skin wound aged 15-30 min, was $65 \%$ for fibronectin and $76 \%$ for P-selectin.

Ohshima (2000) and Ortiz-Rey et al. (2003) concluded that the IHC staining methods are more valuable as the AM skin wound age increases, and also with increasing the PM interval in PM wounds. However in lesions just before or after death, the sensitivity of IHC methods is doubtful.

The current study conducted a receiver operator characteristic (ROC) curve to compare between fibronectin and P-selectin (CD62p) sensitivity and specificity in determining wound vitality and wound age, and confirmed that only in case of fibronectin IHC score 3; the probability that the skin wound sample was from a PM skin wound was $0 \%$. On the other hand, in case of P-selectin IHC score 2 or 3; the probability that the skin sample was from a PM skin wound was $0 \%$, indicating that $\mathrm{P}$-selectin (CD62p) is more reliable and specific than fibronectin as a marker of wound vitality and wound age estimation.

This study found that $40 \%$ of non-injured control samples showed immunopositivity for 
fibronectin and CD62p. Van de Goot et al. (2014) found that $13 \%$ of non-injured control samples exhibited immunopositivity for fibronectin and CD62p, with no clear explanation for this.

In conclusion, the current work confirmed that both P-selectin (CD62p) and fibronectin can be used as markers of the wound vitality and wound age estimation, but CD62p was more reliable and specific than fibronectin.

\section{Recommendations}

- Large scale studies conducted on a large case numbers are needed for better evaluation and comparison between fibronectin and P-selectin as indicators of wound vitality and age estimation.

- Comparing the IHC staining intensity of skin wounds with the same individual normal skin is needed, due to large inter-individual variations of P-selectin and fibronectin expressions.

\section{Acknowledgment}

Sincere thanks and appreciation to all staff members of the Forensic Medicine and Clinical Toxicology Department, Faculty of Medicine, Benha University, for their encouragement and support. http://www.fmed.bu.edu.eg

\section{References}

Balazic, J.; Grajn, A.; Kralj, E.; Serko, A. and Stefanic, B. (2005): "Expression of fibronectin suicidal in gunshot wounds". Foren. Sci. Int., 147:S5-S7.

Betz, P.; Nerlich, A.; Wilske, J.; Tübel, J.; Wiest, I.; Penning, R. and
Eisenmenger, W. (1992):

"Immunohistochemical localization of fibronectin as a tool for the age determination of human skin wounds". Int. J. Leg. Med., 105 (1): 21-26.

Capatina, C.O.; Ceauşu, M.; Curca, C.G.; Tabirca, D.D. and Hostiuc, S. (2012): "Immunophenotypical expression of adhesion molecules in vital reaction". Rom. J. Leg. Med., 20 (3); 185-190.

Capatina, C.O.; Ceauşu, M. and Hostiuc, S. (2013): "Usefulness of Fibronectin and $\mathrm{P}$-selectin as markers for vital reaction in uncontrolled conditions". Rom. J. Leg. Med., 21: 281-286.

Capatina, C.O.; Chirica, V.I.; Martius, E.; Isaila, O.M. and Ceauşu, M. (2015): "Are P-selectin and fibronectin truly useful for the vital reaction? case presentation". Rom. J. Leg. Med., 23: 91-94.

Casse, J.M.; Martrille, L.; Vignaud, J.M. and Gauchotte, G. (2016): "Skin wounds vitality markers in forensic pathology: An updated review". Med. Sci. Law., 56 (2): $128-37$.

Ceauşu, M. and Capatina, C.O. (2014): "Histopathological and immunohistochemical markers in the differential diagnosis of ante-mortem and postmortem traumatic lesions and in the assessment of age of the lesions", Bucharest, Universitary Publishing House.

Cecchi, R. (2010): "Estimating wound age: looking into the future". Int. J. Leg. Med., 124(6):523-536.

Cecchi, R.; D'Annibale, C.; Valente, S. and Ferrera, V. (2004): "La vitalità delle lesioni da arma bianca: studio sperimentale". Zacchia., 77:49- 59. 
Dawson, S. B. and Trapp, R. (1994): Basic and clinical biostatistics. In: Biostatistics: A Foundation for Analysis in The Health Sciences, Lange Medical Book, 2nd ed., prentice - Hall International Inc, chapter (4), P.P. 201-205.

Dressler, J.; Bachmann, L.; Koch, R. and Müller, E. (1999): "Enhanced expression of selectins in human skin wounds". Int. J. Leg. Med., 112: 3944.

Dressler, J.; Bachmann, L.; Strejc, P.; Koch, R. and Müller, E. (2000): "Expression of adhesion molecules in skin wounds: diagnostic value in legal medicine". Foren. Sci. Int., 113:173176.

Dressler, J.; Strejc, P.; Klir, P.; Muller, E.; Boubelik, O. and Grossova, I.(2002): "Time-related expression of adhesive proteins and other markers of age of injuries". Soud. Lek., 47(3): $38-44$.

Fieguth, A.; Feldbrugge, H.; Gerich, T.; Kleemann, W. J. and Troger, H.D. (2003): "The time-dependent expression of fibronectin, MRP8, MRP14 and defensing in surgically treated human skin wounds". Foren. Sci. Int., 131: 156-161.

Fieguth, A.; Kleemann, W.J.; Von, W.R.; Werner, M. and Troger, H.D.(1997): "Influence of postmortem changes on immunohistochemical reactions in skin". Int. J. Leg. Med., 110 (1): 18-21.

Greenberg, R.S.; Daniels, R.S.; Flanders, W.D.; Eley, J.W. and Boring, J.R. (1996): Diagnostic testing. In: Medical Epidemiology, $3^{\text {rd }}$ edition. McGraw-Hill, New York, P.P. 77-89.
Grellner, W.; Dimmeler, S. and Madea, B. (1998): "Immunohistochemical detection of fibronectin in postmortem incised wounds of porcine skin". Foren. Sci. Int., 97(2-3):109-116.

Grellner, W. and Madea, B. (2007): "Demands on scientific studies: vitality of wounds and wound age estimation". Foren. .Sci. Int., 165 (2-3):150-154.

Hayashi, T.; Ishida, Y.; Kimura, A.; Takayasu, T.; Eisenmenger, W. and Kondo, T. (2004): "Forensic application of VEGF expression to skin wound age determination". Int. J. Leg. Med., 118: 320-325.

Huang, P.; Yang, G.D.; Tuo, Y. and Wang, Z.Y. (2007): "The analysis of vital reaction following skin injury close to death in mouse". FTIR. Spectrosc. Spectral Anal., 27: 1074-1076.

Ishida, Y.; Kimura, A.; Takayasu, T.; Eisenmenger, $W$. and Kondo, $T$. (2009): "Detection of fibrocytes in human skin wounds and its application for wound age determination". Int. J. Leg. Med., 123: 299-304.

Kondo, T. (2007): "Timing of skin wounds". Leg. Med., 9:109-114.

Ohshima, T. (2000): "Forensic wound examination". Foren. Sci. Int., 113 (1): 153-164.

Ortiz-Rey, J.A.; Suárez-Peñaranda, J.M.; Muñoz-Barús, J.I.; Alvarez, C.; San Miguel, P.; Rodríguez-Calvo, M.S. and Concheiro-Carro, L. (2003): "Expression of fibronectin and tenascin as a demonstration of vital reaction in rat skin and muscle". Int. J. Leg. Med., 117: 356-60.

Ortiz-Rey, J.A.; Suárez-Peñaranda, J.M.; San Miguel, P.; Muñoz, J.I.; 
Rodríguez-Calvo, M.S. and Concheiro, L. (2008): "Immunohistochemical analysis of P-Selectin as a possible marker of vitality in human cutaneous wounds".J. Foren. Leg. Med., 15(6): 368-372.

Van de Goot, F.R.W. and Fronczek, J. (2017): The dating of Injury; The five step approach. A clinical method to Enlighten forensic aspects. In: Essentials of Autopsy Practice, Reviews,
Updates, and Advances". Springer International Publishing AG, chapter (7), P.P. 157-177.

Van de Goot, F.R.W.; Korkmaz, H.I.; Fronczek, J.; et al. (2014): "A new method to determine wound age in early vital skin injuries: a probability scoring system using expression levels of fibronectin, CD62p and factor VIII in wound hemorrhage". Foren. Sci. Int., 244: $128-135$.. 


\title{
دقة الفيبرونيكتن و البى سيلكتين كمؤشرات على حيوية و عمر الجروح: دراسة مناعية هستوكميائية على جروح الجلد في الأنسان
}

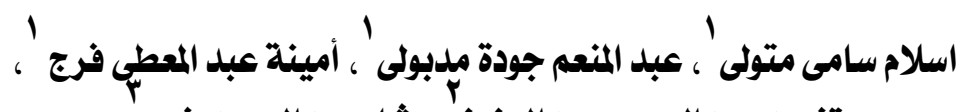

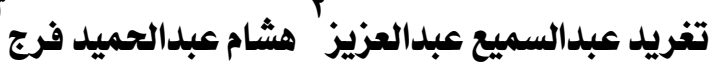

'الطب الثرعى و السموم الأكلينيكية، 'الباثولوجى،كلية الطب، جامعة بنها، مصر،

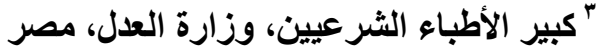

الفيبرونيكنن و البي سيلكتين من المؤشرات المناعية التى نشير الى حيويـة و كذلك عمر الجروح الجلديـة.

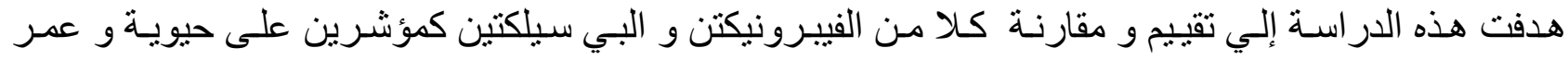

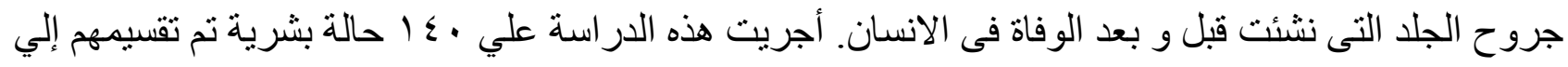

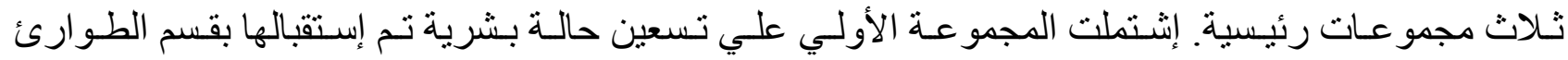

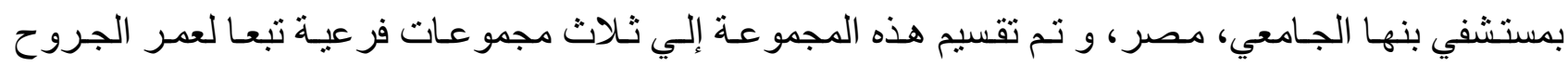

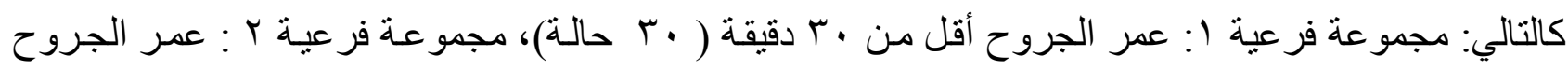

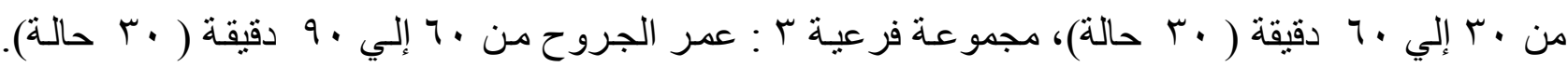

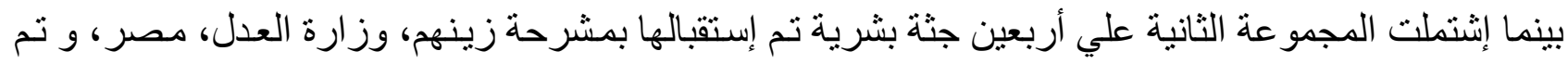

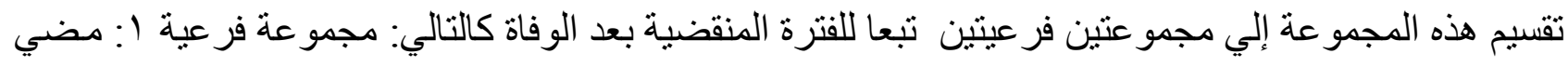

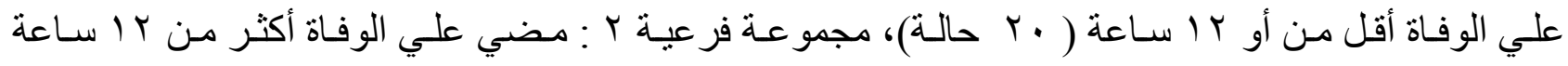
(· • حالة). و إشتملت المجمو عة الثالثة علي عشر عينات من جلد بشري طبيعي للمقارنة بها. تم إستخدام التقنية

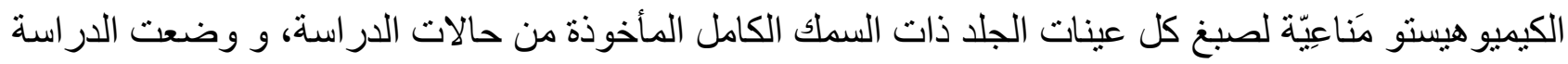
نظام نقاط لتحديد العلاقة بين عمر الجروح و زيادة كثافة الصبغة المناعية المستخدمة سواء الفيبرونيكتن أو البي

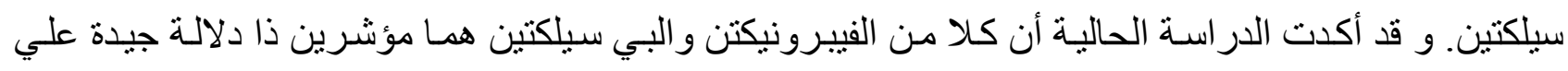

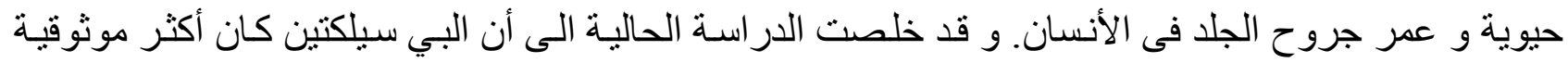

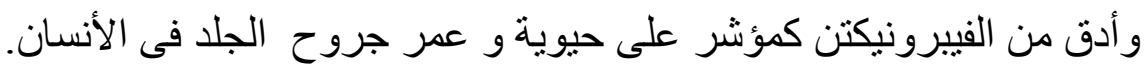

